\title{
Reversible Switching Phenomenon in Diarylethene Molecular Devices with Reduced Graphene Oxide Electrodes on Flexible Substrates
}

\author{
Dongku Kim, Hyunhak Jeong, Wang-Taek Hwang, Yeonsik Jang, Dmytro Sysoiev, \\ Elke Scheer, Thomas Huhn, Misook Min,* Hyoyoung Lee, and Takhee Lee*
}

\begin{abstract}
Photoswitching molecular electronic devices with reduced graphene oxide (rGO) top electrodes on flexible substrates are fabricated and characterized. It has been reported previously that diarylethene molecular devices with poly-(3,4-ethylenedioxythiophene) stabilized with poly-(4-styrenesulfonic acid)/Au top electrodes can hold two stable electrical conductance states when the devices are exposed to UV or visible light during device fabrication. However, those devices fail to show the reversible switching phenomenon in response to illumination after device fabrication. By employing conducting and transparent rGO top electrodes, it is demonstrated that the diarylethene molecular devices show a reversible switching phenomenon, i.e., the fabricated devices change their conductance state in response to the alternating illumination with UV and visible light. Furthermore, the molecular devices with rGO top electrodes also exhibit good longtime stability and reliable electrical characteristics when subjected to various mechanical stresses (bending radius down to $5 \mathrm{~mm}$ and bending cycle over $10^{4}$ ).
\end{abstract}

\section{Introduction}

Molecular electronic devices, which use single molecules or a molecular monolayer as an active electronic device component, have been widely researched in many device categories

D. Kim, H. Jeong, W.-T. Hwang, Y. Jang, Dr. M. Min,

Prof. T. Lee

Department of Physics and Astronomy

and Institute of Applied Physics

Seoul National University

Seoul 151-747, South Korea

E-mail:minimi0924@gmail.com; tlee@snu.ac.kr

Dr. D. Sysoiev, Dr. T. Huhn

Department of Chemistry

University of Konstanz

78457 Konstanz, Germany

Prof. E. Scheer

Department of Physics

University of Konstanz

78457 Konstanz, Germany

Prof. H. Lee

Center for Integrated Nanostructure Physics

Institute for Basic Science (IBS)

Department of Energy Science

Department of Chemistry

Sungkyunkwan University

Suwon 440-746, South Korea such as rectifiers, transistors, memories, and photoswitches. ${ }^{[1-18]}$ However, molecular devices suffer from a very low device yield because it is difficult to attach metal electrodes on a molecular size scale. For example, in a vertical metal-moleculemetal junction structure, the metal deposited in the top electrode penetrates into the existing molecular monolayer to the bottom electrode, thus causing problems with electrical short circuits and a typical device yield of less than $\approx 1 \% \cdot{ }^{[19-21]}$ To resolve the low-yield problems, intermediate layers of conducting polymer ${ }^{[2-25]}$ or graphene film ${ }^{[12,13,26,27]}$ have been introduced that greatly enhance the device yield and reliability. ${ }^{[26]}$

By employing poly-(3,4-ethylenedioxythiophene) stabilized with a poly(4-styrenesulfonic acid) (PEDOT:PSS) interlayer in the molecular devices, we have previously demonstrated high device yield and reliable initialization of the photostate in molecular devices of the diarylethene class on flexible substrates. ${ }^{[18,22]}$ The diarylethene photoswitching molecular devices have shown two stable electrical states; a closed (high conductance) state or an open (low conductance) state are created upon illumination with UV or visible light, respectively. ${ }^{[6,28-31]}$ However, these two conductance states were defined and fixed during the device fabrication with illumination of either UV or visible light and the devices failed to show the reversible switching phenomenon. ${ }^{[18]}$ This failure of reversible photoswitching could be caused by the low transmittance of light through the PEDOT:PSS/Au top electrode layer, low power intensity of the UV or visible handed lamp, or a combination of these effects. Without the reversible switching phenomenon, the photoswitching molecular devices cannot be considered to be true switching devices.

We fabricated and characterized reversible photoswitching molecular electronic devices composed of the diarylethene class but by using reduced graphene oxide (rGO) top electrodes and mounted on plastic substrates. The photoswitching molecular devices with an rGO top electrode clearly showed two stable electrical states with different current levels. To test the flexibility, we examined the electrical characteristics of both states under bending conditions with various bending radii (down to $5 \mathrm{~mm}$ ) and bending cycles (up to 1000 times). More importantly, 
we examined and successfully observed a reversible switching phenomenon in fabricated molecular devices with an rGO top electrode under ambient conditions.

\section{Results and Discussion}

Figure 1a shows schematics that illustrate the fabrication processes for the photoswitching molecular electronic devices on a flexible substrate. The fabrication process is similar to our previously reported one, ${ }^{[18]}$ except for changes to the top electrode identity and a few processing steps. First, we deposited Au $(60 \mathrm{~nm}) / \mathrm{Ti}(5 \mathrm{~nm})$ on a polyethylene terephthalate (PET) flexible substrate by using an electron-beam (e-beam) evaporator at a slow deposition rate of $\approx 0.1 \AA \mathrm{s}^{-1}$ to obtain a smooth surface for the bottom electrodes. Then, a self-assembled monolayer (SAM) of the photoswitching molecules was deposited on the exposed Au surfaces. The photoswitching molecule was a diarylethene species, the chemical structure of which is shown in Figure 1c. The photoswitching device has two different electrical conductance states, which are identified as the closed (high degree of $\pi$-conjugation) state or the open (broken $\pi$-conjugation) state. ${ }^{[28]}$ It is possible to convert from the open to the closed state by exposing the molecules to UV light (at wavelengths from 290 to $370 \mathrm{~nm}$ ), or from the closed to the open state with visible light (at wavelengths from 450 to $630 \mathrm{~nm}) .{ }^{[28,29,32-34]}$ For the SAM deposition process, we prepared a diluted solution of the molecules $\left(\approx 3 \times 10^{-3} \mathrm{M}\right)$ in ethanol and added a small amount of ammonium hydroxide $\left(\mathrm{NH}_{4} \mathrm{OH}\right)$ to the molecular solution in order to deprotect the thiol end-group from the acetyl $\left(\mathrm{COCH}_{3}\right)$, so that the thiol end-group enables good electrical contact to the $\mathrm{Au}$ electrodes by thiol-Au chemisorption. ${ }^{[35-37]}$
Then, we immersed the samples in this molecular solution for $1-2 \mathrm{~d}$ in an $\mathrm{N}_{2}$-filled glove box. After finishing the SAM deposition, rGO in dimethylformamide (DMF) was spin-coated twice over the SAMs to protect them from any outside contaminants. Next, the rGO top electrode was made by spraying rGO solutions through a shadow mask with a side length from 200 to $600 \mu \mathrm{m}$. The samples were exposed to a visible $(520 \mathrm{~nm})$ laser for $\approx 30 \mathrm{~min}$ or to a UV $(360 \mathrm{~nm})$ laser for $\approx 1 \mathrm{~h}$ to be defined as the open state or closed state, respectively, during the device fabrication. More detailed information regarding the device fabrication procedures is given in the Experimental Section and in Figure S1 in the Supporting Information. Figure 1b shows a series of optical, scanning electron microscopy (SEM), and transmission electron microscopy (TEM) images of the fabricated photoswitching molecular devices on flexible substrates. In the TEM images, we can identify each layer consisting of Ti, $\mathrm{Au}$, and C (see Figure S2 in the Supporting Information).

First, we examined whether rGO films can be used as good transparent and conducting electrodes for the photoswitching molecular devices on a plastic substrate. Figure 2 shows the transmittance, current-voltage graph, Raman spectra, and X-ray photoelectron spectroscopy (XPS) data for an rGO film on a glass substrate. Figure 2a shows transmittance plots for an rGO film and a PEDOT:PSS/Au film. Previously, we used a PEDOT:PSS/Au film as the top electrode for photoswitching molecular devices, but those devices failed to show the reversible photoswitching phenomenon. ${ }^{[18]}$ The absence of reversible photoswitching in the molecular devices with PEDOT:PSS/Au top electrodes could be caused by the relatively low transmission characteristics of PEDOT:PSS/Au compared to those for an rGO film. We also checked the conductivity of the rGO film. For this, we fabricated a pattern with an rGO film in contact with two Au

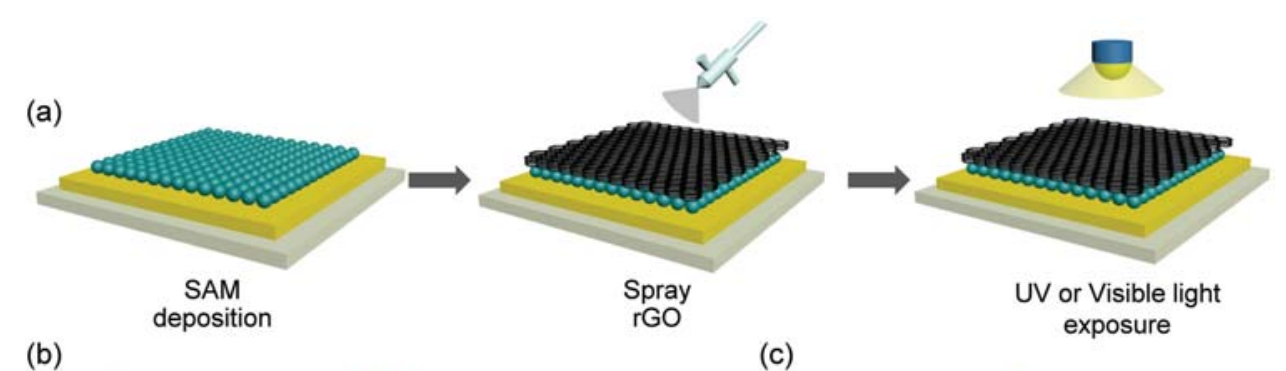

(b)

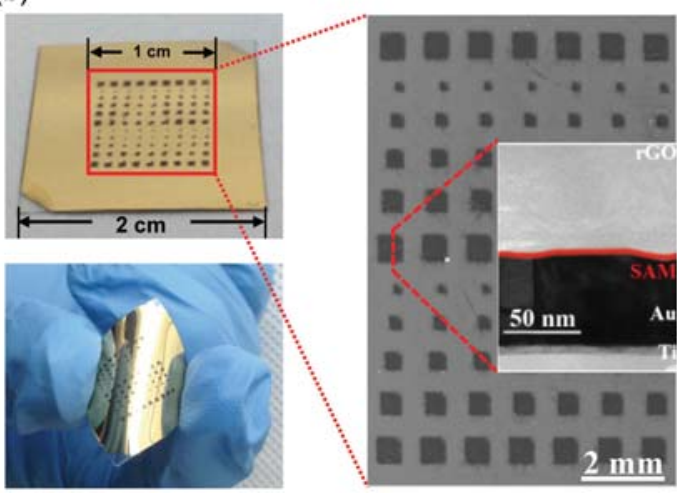

(c)

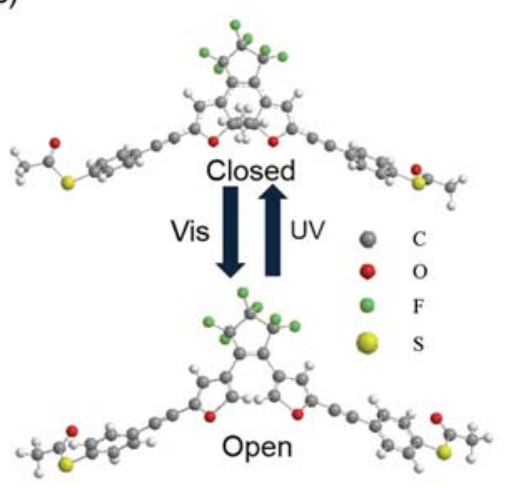

Figure 1. a) Schematics illustrating the device fabrication processes for photoswitching molecular devices with an rGO top electrode on a flexible substrate. b) Optical, SEM, and cross-sectional TEM images of the photoswitching molecular devices. The molecular junctions are squares with a side length ranging from 200 to $600 \mu \mathrm{m}$. c) Chemical structures of the diarylethene. The closed state (high conductance) can be changed to the open state with UV irradiation whereas the open state (low conductance) can be changed to the closed state by illumination with visible light. 
(a)

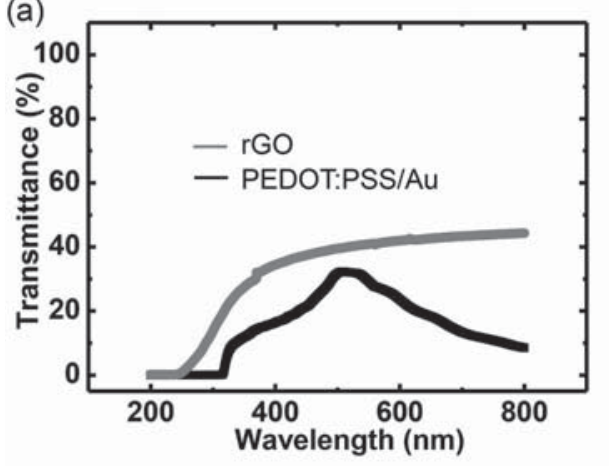

(c)

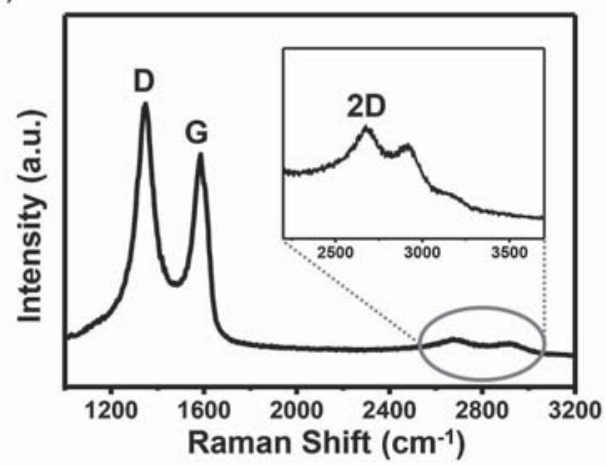

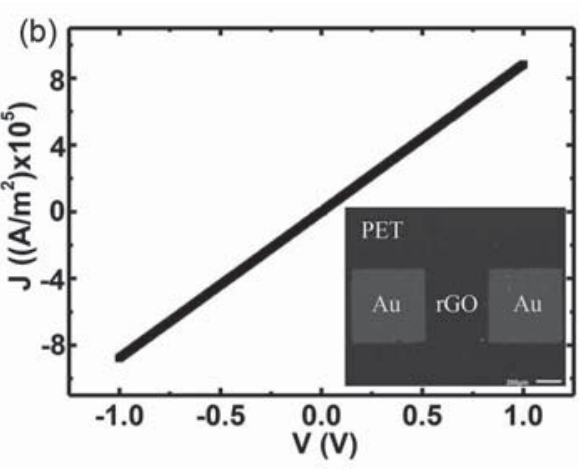

(d)

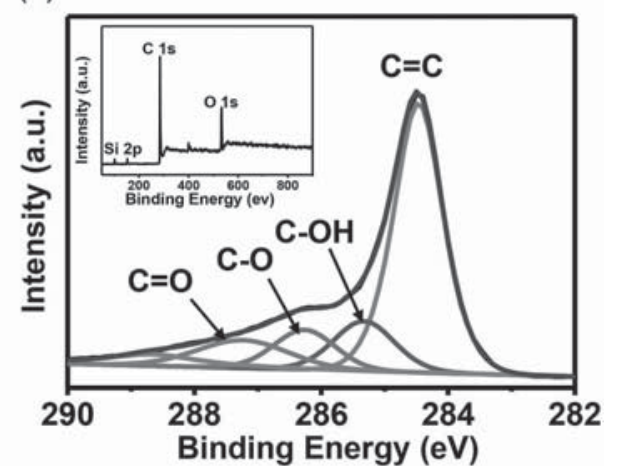

Figure 2. a) The transmittance of $\mathrm{rGO}$ and PEDOT:PSS/Au films on glass substrates. b) J-V plot of an rGO film pattern in contact with Au electrodes. c,d) Raman spectroscopy and XPS data of an rGO film.

electrodes (see inset of Figure $2 \mathrm{~b}$ ) and measured the current density-voltage $(J-V)$ characteristics. The results are shown in Figure $2 \mathrm{~b}$. From this $J-V$ plot, we found the conductivity of an rGO film to be $3700 \mathrm{~S} \mathrm{~m}^{-1}$. [38] The results of Figure 2a,b indicate that an rGO film can be used as the top electrode in photoswitching devices. ${ }^{[12,13]}$ We observed the typical G, D, and 2D peaks that were located at 1590, 1350, and $2910 \mathrm{~cm}^{-1}$, respectively. It is well known that G-mode is a primary in-plane vibrational mode, indicating $\mathrm{C}-\mathrm{C} \mathrm{sp}^{3}$ stretching. The D-mode is a first or second order overtone for a different in-plane vibration, indicating a structural defect in the crystal where $\mathrm{C} \equiv \mathrm{C} \mathrm{sp}{ }^{2}$ is created because of $\mathrm{C}-\mathrm{C} \mathrm{sp}{ }^{3}$ bond formation. ${ }^{[39,40]}$ The XPS data of rGO are shown in Figure 2d. The XPS peak at $284.5 \mathrm{eV}$ (C 1s peak) is assigned to the carbon atoms in $\mathrm{C}=\mathrm{C}$ bond. XPS peaks at 285.3, 286.3, and $287.3 \mathrm{eV}$ correspond to $\mathrm{C}-\mathrm{OH}, \mathrm{C}-\mathrm{O}$, and $\mathrm{C}=\mathrm{O}$, respectively. ${ }^{[1,42]}$ The XPS results indicate that graphene oxide was reduced enough to be used as top electrode.

Figure $3 \mathrm{a}$ shows the $J-V$ data from the diarylethene molecular devices on flexible substrates in the open and closed states under a flat substrate condition (having a bending radius $=\infty$ ). We measured the electrical characteristics of our molecular devices by probing the electrodes of the devices in a probe station (Figure 3a inset). The open and closed states were defined and fixed during device fabrication. The error bar indicates the standard deviation obtained from measuring many devices $(\approx 20$ devices at each state condition). We confirmed that the current of the closed state was higher than that of the open state, as has been observed for the molecular junctions with PEDOT:PSS/Au top electrode. ${ }^{[6,18]}$ There are three mechanisms resulting in the different electrical currents of the closed and open states. ${ }^{[28,29]}$ First, when the open state is exposed to UV light, the open state changes to the closed state by forming a completely $\pi$-conjugated system (see Figure 1c). Equivalently, when the closed state is exposed to visible light, the closed state changes to the open state and the $\pi$-conjugation is broken (see Figure 1c). As a result, the energy gap between the highest occupied molecular orbital (HOMO) and the lowest unoccupied molecular orbital (LUMO) of the closed state is smaller than that of the open state. In addition, in the transport experiments of similar diarylethene molecules, the level broadening of the current-carrying molecular orbital of the closed state was found to be bigger than that in the open state, although the level alignment between the current-carrying molecular orbital and the Fermi energy of the Au electrodes was found to be better in the open state than in the closed state. ${ }^{[28,29]}$ As a net result, the current of the closed state is higher than that of the open state.

To investigate the stability and reliability of our photoswitching molecular devices with an rGO top electrode, we measured the current density $(J)$ at $+0.8 \mathrm{~V}$ for the closed and open state under flat (as defined $r=\infty$ ) conditions for up to $10^{4} \mathrm{~s}$ in increments of $100 \mathrm{~s}$ (Figure 3b). Additionally, we measured the $J$ values for alternating voltages of $\pm 0.8 \mathrm{~V}$ under flat conditions for up to $10^{4} \mathrm{~s}$ with an increment of $10 \mathrm{~s}$ (Figures 3c,d). The graphs of Figure 3 indicate that photoswitching molecular devices with an rGO top electrode do not show any noticeable degradation during the retention measurements for the two electrical states. Moreover, we measured the $J$ values of the closed and open states after the devices were stored for $30 \mathrm{~d}$ 

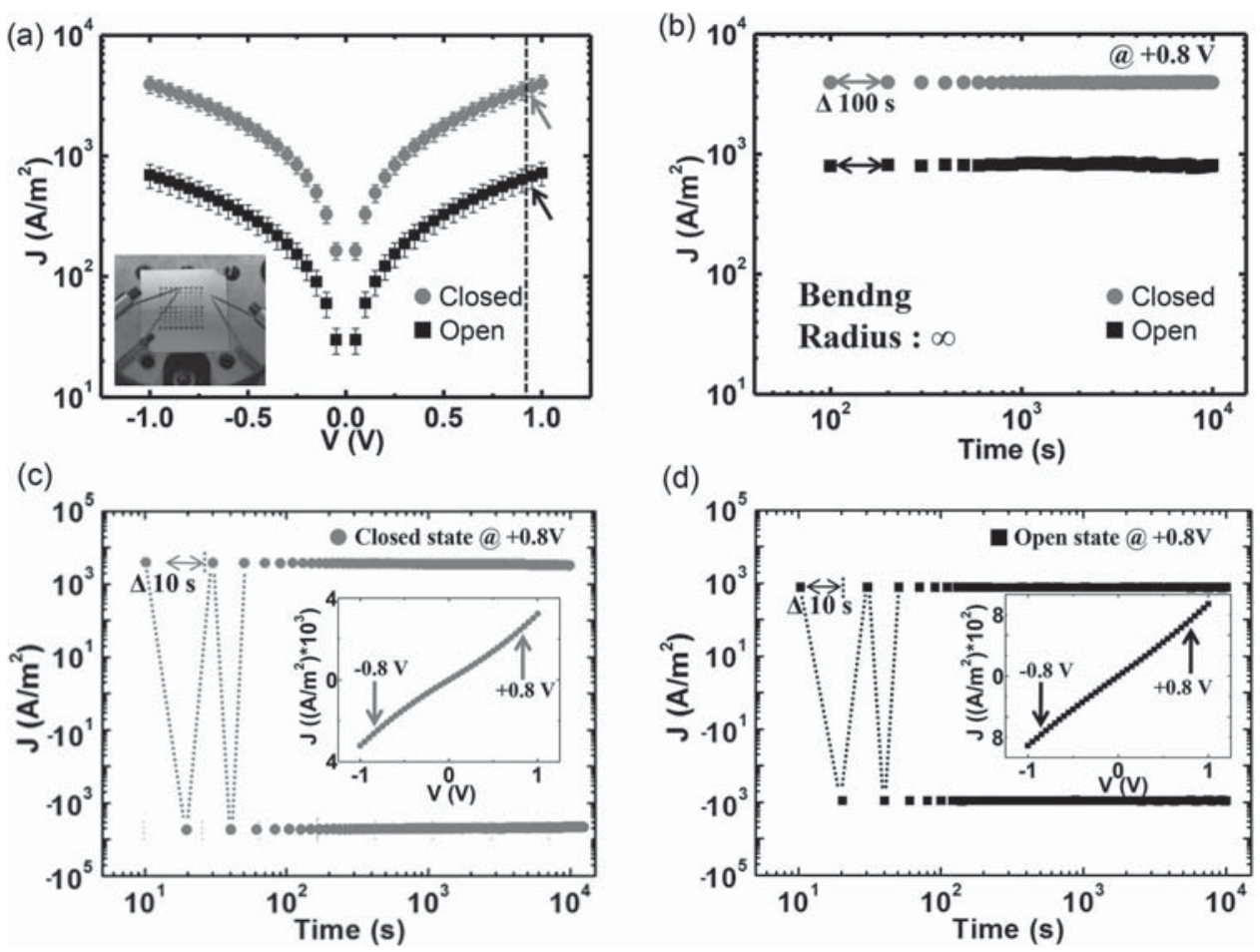

Figure 3. a) $J-V$ curves for the closed and open state diarylethene molecular devices with an $\mathrm{CGO}$ top electrode on a PET substrate under flat conditions. Red and black arrows indicate the $J$ values measured at $0.8 \mathrm{~V}$ (shown by a dashed line). b) Retention characteristics for the closed and open states, showing $J$ values measured at $0.8 \mathrm{~V}$ for up to $10^{4} \mathrm{~s}$ with an increment of $100 \mathrm{~s}$. c,d) Endurance characteristics of c) the closed and d) open states observed under two step voltage conditions. The $J$ values of the closed and open states were measured at $\pm 0.8 \mathrm{~V}$ for up to $10^{4} \mathrm{~s}$ with an increment of $10 \mathrm{~s}$. The inset plot in (c,d) shows the $J-V$ curves prior to these measurements for the closed and open states.

under ambient conditions. We did not observe any serious degradation of the devices in this test, either (see Figures S6a,b in the Supporting Information). All of these results indicate that photoswitching molecular devices with an rGO top electrode can maintain good electrical properties for a long period of time.

To investigate the photoswitching molecular devices with an rGO top electrode under mechanical deformation, we measured the $J$ values of the closed and open states under several bending conditions. Figure $4 \mathrm{a}, \mathrm{b}$ shows the $J$ values at $+0.8 \mathrm{~V}$ for the closed and open states under bending cycles $(0,10,100$, and 1000 times) and bending radii $(\infty, 10$, and $5 \mathrm{~mm})$.

The complete $J-V$ data sets and selected photographs of our automatic cyclical bending test machine are provided in Figures S4 and S7 in the Supporting Information. Additionally, we measured the $J$ values for the closed and open states for up to $10^{4}$ seconds under a $5 \mathrm{~mm}$ bending radius condition (Figure 4c), indicating that the photoswitching molecular devices with an rGO top electrode maintained consistent $J$ values with different current levels for each state during the retention measurements. The results presented in Figure 4 demonstrate that our devices on the flexible substrates were operated reliably and with good stability under the various bending conditions.

Lastly and importantly, we examined the reversible photoswitching phenomenon in our molecular devices with an rGO top electrode. The photoswitching devices should reversibly switch between the two electrical states in response to the light illumination. Figure 5a shows the $J-V$ plots for the initial closed states (red circles), $J-V$ plots for conversion to the open states after exposure to visible light (black rectangles, from the closed to open state), and $J-V$ plots for conversion to the closed states after exposure to UV (blue triangles, from the open to closed state). The error bars represent the standard deviation obtained from measuring $\approx 20$ devices during the photoswitching conversion. The current levels of the photoswitchable open and closed states were consistent when compared to the data in Figure 3a. We measured the reversible photoswitching phenomenon 20 times by alternating between irradiation with a UV and a visible laser; the results are shown in Figure 5b. The complete $J-V$ data sets for the reversible photoswitching processes are provided in Figure S5 in the Supporting Information. In addition, we measured the reversible photoswitching phenomenon after the devices were stored for $30 \mathrm{~d}$ in ambient conditions (see Figure S6c in the Supporting Information). With these results, we were able to demonstrate that the diarylethene molecular devices with an rGO top electrode exhibited a reliable and reversible photoswitching phenomenon in response to illumination.

\section{Conclusion}

We have fabricated photoswitching molecular devices of the diarylethene class with an rGO top electrode on a PET flexible substrate. The photoswitching diarylethene molecules have 
(a)

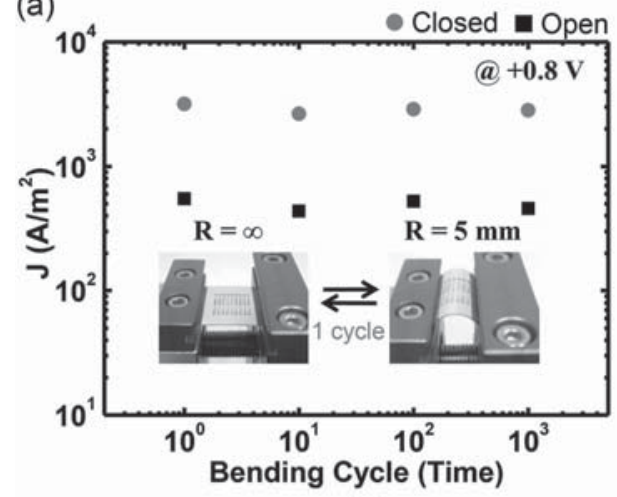

(b)

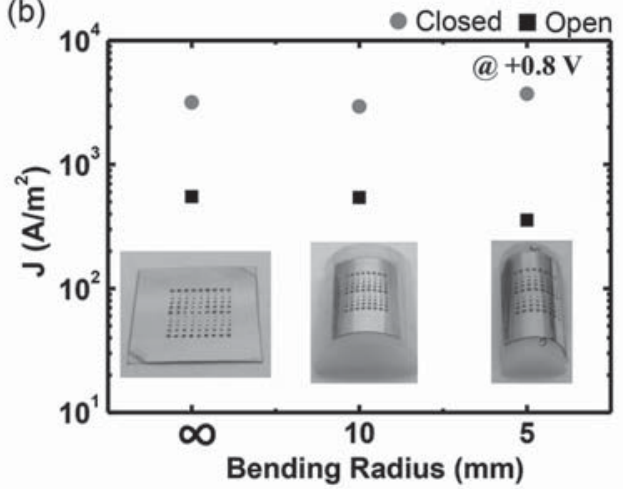

(c)

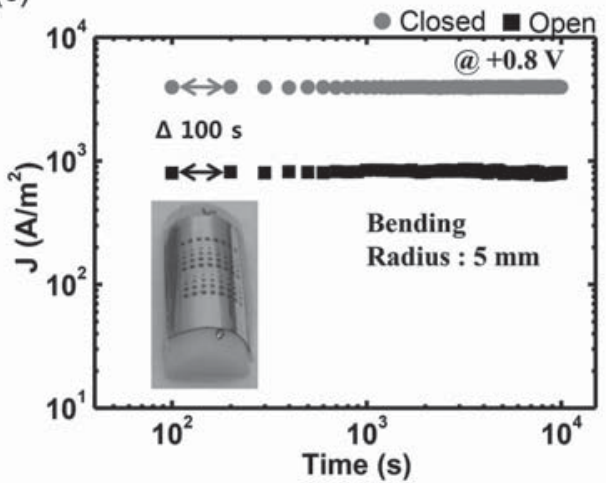

Figure 4. a) $J$ values measured at $0.8 \mathrm{~V}$ for the closed and open states after being subjected to repeated bending cycles $(0,10,100$, and 1000 times). Inset photographs show one bending cycle at a bending radius of $5 \mathrm{~mm}$. b) J values measured at $0.8 \mathrm{~V}$ for the closed and open states under the flat substrate condition (bending radius $=\infty$ ) and different bending conditions (bending radii of $10 \mathrm{~mm}$ and $5 \mathrm{~mm}$ ). Inset photographs show the molecular devices under different bending radius configurations. c) Retention characteristics for the closed and open states under severe bending conditions (bending radius of $5 \mathrm{~mm}$ ). The inset shows a molecular device that was wound around a half cylindrical rod with a bending radius of $5 \mathrm{~mm}$.
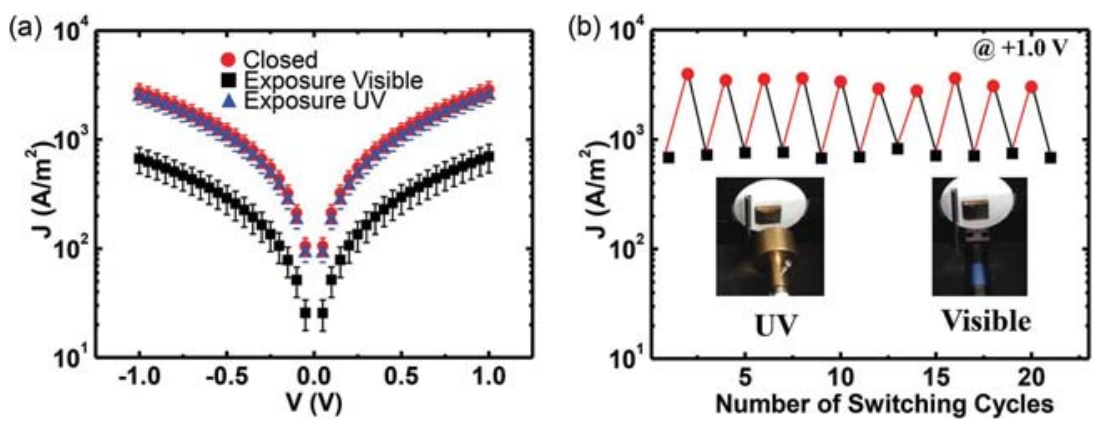

Figure 5. a) $J-V$ curves of the photoswitching molecular devices at initial closed states (red circles), open states that were converted from the closed states with visible light illumination (black rectangles), and closed states that were converted from the open states with UV illumination (blue triangles). b) The reversible photoswitching phenomenon repeated 20 times by alternating UV and visible light irradiation.

two electrical conductance states: a high conductance and a low conductance state, established by UV or visible light irradiation. Unlike the molecular devices with PEDOT:PSS/Au top electrodes that failed to show the reversible photoswitching phenomenon, we successfully demonstrated the reliable and reversible photoswitching phenomenon in molecular devices with rGO top electrodes upon exposure to UV or visible light. Additionally, the electrical properties of our photoswitching molecular devices with an rGO top electrode were well maintained under various mechanical deformations. This study may aid in the development of electrical or optical functional molecular devices on flexible substrates.

\section{Experimental Section}

Fabrication of Photoswitching Molecular Devices with an rGO Top Electrode on Flexible Substrates: After the flexible substrates were cleaned in isopropyl alcohol and deionized water, the patterned bottom Au $(60 \mathrm{~nm}) / \mathrm{Ti}(5 \mathrm{~nm})$ electrodes were deposited on the PET flexible substrates by using an e-beam evaporator. The SAM deposition of diarylethene molecules was achieved by dipping the flexible substrates into the SAM solutions $\left(\approx 3 \times 10^{-3} \mathrm{M}\right.$ in anhydrous ethanol) in an $\mathrm{N}_{2}$-filled glove box for $\approx 1 \mathrm{~d}$. After deposition of SAMs, the flexible substrates were rinsed with anhydrous ethanol and then dried in an $\mathrm{N}_{2}$-filled glove box for $\approx 2 \mathrm{~h}$. Next, $0.5 \mathrm{mg} \mathrm{mL}^{-1}$ of $\mathrm{rGO}$ in DMF was spin-coated over the SAMs on flexible substrates at $3500 \mathrm{rpm}$ for $30 \mathrm{~s}$ to protect the molecular layer from outside contaminants. Then, the rGO top electrode was fabricated by spraying rGO solutions on a shadow mask with a side length from 200 to $600 \mu \mathrm{m}$. The photoswitching molecular devices with an rGO top electrode can be changed from the 
open to the closed state when exposed to UV $(360 \mathrm{~nm})$ and from the closed to open state when exposed to a visible $(520 \mathrm{~nm})$ laser.

Device Characterization: The electrical characteristics of the molecular devices were measured with a semiconductor parameter analyzer (Keithley 4200 SCS) and a probe station system (JANIS Model ST-500) under ambient conditions.

\section{Supporting Information}

Supporting Information is available from the Wiley Online Library or from the author.

\section{Acknowledgements}

The authors appreciate the financial support of the National Creative Research Laboratory program (Grant No. 2012026372) through the Korean National Research Foundation (NRF) funded by the Korean Ministry of Science, ICT, and Future Planning. E.S. and T.H. thank the Deutsche Forschungsgemeinschaft through SFB767.

[1] J. Chen, M. A. Reed, A. M. Rawlett, J. M. Tour, Science 1999, 286, 1550.

[2] W. Liang, M. P. Shores, M. Bockrath, J. R. Long, H. Park, Nature 2002, 417, 725.

[3] J. Park, A. N. Pasupathy, J. I. Goldsmith, C. Chang, Y. Yaish J. R. Petta, M. Rinkoski, J. P. Sethna, H. D. Abruna, P. L. McEuen, D. C. Ralph, Nature 2002, 417, 722.

[4] J. E. Green, J. W. Choi, A. Boukai, Y. Bunimovich, E. Johnston-Halperin, E. Delonno, Y. Luo, B. A. Sheriff, K. Xu Y. S. Shin, H. R. Tseng, J. F. Stoddart, J. R. Heath, Nature 2007, 445, 414.

[5] T. Kudernac, T. Kobayashi, A. Uyama, K. Uchida, S. Nakamura, B. L. Feringa, J. Phys. Chem. A. 2013, 117, 8222.

[6] A. J. Kronemeijer, H. B. Akkerman, T. Kudernac, B. J. van Wees, B. L. Feringa, P. W. M. Blom, B. de Boer, Adv. Mater. 2008, 20, 1467.

[7] H. Song, Y. Kim, Y. H. Jang, H. Jeong, M. A. Reed, T. Lee, Nature 2009, 462, 1039.

[8] C. A. Nijhuis, W. F. Reus, A. C. Siegel, G. M. Whitesides, J. Am. Chem. Soc. 2011, 133, 15397.

[9] M. Del Valle, R. Gutierrez, C. Tejedor, G. Guniberti, Nat. Nanotechnol. 2007, 2, 176.

[10] K. Mastsuda, M. Irie, J. Am. Chem. Soc. 2001, 123, 9896.

[11] S. J. van der Molen, J. Liao, T. Kudernac, J. S. Agustsson, L. Bernard, M. Calame, B. J. van wees, B. L. Feringa, C. Schönenberger, Nano Lett. 2009, 9, 76.

[12] S. Seo, M. Min, S. M. Lee, H. Lee, Nat. Commun. 2013, 4, 1920.

[13] M. Min, S. Seo, S. M. Lee, H. Lee, Adv. Mater. 2013, 24, 7045.

[14] F. Pump, G. Cuniberti, Surf. Sci. 2007, 601, 4109.

[15] H. Jeong, D. Kim, G. Wang, S. Park, H. Lee, K. Cho, W.-T. Hwang, M.-H. Yoon, Y. H. Jang, H. Song, D. Xing, T. Lee, Adv. Funct. Mater. 2014, 24, 2472.

[16] Y. Li, H. Zhang, C. Qi, X. Guo, J. Mater. Chem. 2012, 22, 4261.
[17] C. Jia, J. Wang, C. Yao, Y. Cao, Y. Zhong, Z. Liu, Z. Liu, X. Guo, Angew. Chem. Int. Ed. 2013, 52, 8666.

[18] D. Kim, H. Jeong, H. Lee, W.-T. Hwang, J. Wolf, E. Scheer, T. Huhn, H. Jeong, T. Lee, Adv. Mater. 2014, 26, 3968.

[19] T. W. Kim, G. N. Wang, H. Lee, T. Lee, Nanotechnology 2007, 18 , 315204.

[20] G. Wang, T. W. Kim, H. Lee, T. Lee, Phys. Rev. B 2007, 76, 205320.

[21] G. Wang, H. Yoo, S. I. Na, B. Cho, D. Y. Kim, T. Lee, Thin Solid Films 2009, 518, 824.

[22] H. B. Akkerman, P. W. M. Blom, B. de Boer, Nature 2006, 441, 69.

[23] P. A. Van Hal, E. C. P. Smits, T. C. T. Geuns, H. B. Akkerman, B. C. De Brito, S. Perissinotto, G. Lanzani, A. J. Kronemeijer V. Geskinm, J. Cornil, P. W. Blom, B. de Boer, D. M. de Leeuw, Nat. Nanotechnol. 2008, 3, 7849 .

[24] A. B. Neuhausen, A. Hosseini, J. A. Sulpizio, C. E. D. Chidsey, D. Goldhaber-Gordon, ACS Nano 2012, 6, 9920.

[25] S. Park, G. Wang, B. Cho, Y. Kim, S. Song, Y. Ji, M. H. Yoon, T. Lee, Nat. Nanotechnol. 2012, 7, 438.

[26] G. Wang, Y. Kim, M. Choe, T. W. Kim, T. Lee, Adv. Mater. 2011, 23, 755.

[27] S. Seo, M. Min, J. Lee, T. Lee, S. Y. Choi, H. Lee, Angew. Chem. Int. Ed. 2012, 51, 108

[28] Y. Kim, T. J. Hellmuth, D. Sysoiev, F. Pauly, T. Pietsch, J. Wolf, A. Erbe, T. Huhn, U. Groth, U. E. Steiner, E. Scheer, Nano Lett. 2012, 12, 3736.

[29] T. Sendler, K. Luka-Guth, M. Wieser, Lokamani, J. Wolf, M. Helm, S. Gemming, J. Kerbusch, E. Scheer, T. Huhn, A. Erbe, Adv. Sci. 2015, 2, 1500017 .

[30] N. Katsonis, T. Kudernac, M. Walk, S. J. van der Molen, B. J. van Wees, B. L. Feringa, Adv. Mater. 2006, 18, 1397.

[31] D. Dulic, S. J. van der Molen, T. Kudernac, H. T. Jonkman J. J. D. de Jong, T. N. Bowden, J. van Esch, B. L. Feringa, B. J. van Wees, Phys. Rev. Lett. 2003, 91, 207402.

[32] D. Sysoiev, A. Fedoseev, Y. Kim, T. E. Exner, J. Boneberg, T. Huhn, P. Leiderer, E. Scheer, U. Groth, U. E. Steiner, Chem. Eur. J. 2011, 17, 6663.

[33] D. Sysoiev, T. Yushchenko, E. Scheer, U. Groth, U. E. Steiner, T. E. Exner, T. Huhn, Chem. Commun. 2012, 48, 11355.

[34] J. Wolf, I. Eberspächer, U. Groth, T. Huhn, J. Org. Chem. 2013, 78, 8366.

[35] J. M. Tour, L. Jones II, D. L. Pearson, J. J. S. Lamba, T. P. Burgin, C. M. Whitesides, D. L. Allara, A. N. Parkih, S. V. Atre, J. Am. Chem. Soc. 1995, 117, 9529.

[36] J. G. Kushmerick, J. Lazorcik, C. H. Patterson, R. Shashidhar, D. S. Seferos, G. C. Bazan, Nano Lett. 2004, 4, 639.

[37] H. Valkenier, E. H. Huisman, P. A. van Hal, D. M. de Leeuw, R. C. Chiechi, J. C. Hummelen, J. Am. Chem. Soc. 2011, 133, 4930.

[38] S. Seo, M. Min, J. Lee, T. Lee, S.-Y. Choi, H. Lee, Angew. Chem. Int. Ed. 2012, 41, 108.

[39] H. Zhang, E. Bekyarova, J. W. Huang, Z. Zhao, W. Bao, F. Wang, R. C. Haddon, C. N. Lau, Nano Lett. 2011, 11, 4047.

[40] A. C. Ferrari, J. C. Meyer, V. Scardaci, C. Casirahi, M. Lazzeri, F. Mauri, S. Piscanec, D. Jiang, K. S. Novoselov, S. Roth, A. K. Geim, Phys. Rev. Lett. 2006, 97, 187401.

[41] E. Bekyarova, M. E. Itkis, P. Ramesh, C. Berger, M. Sprinkle, W. A. de Heer, R. C. Haddon, J. Am. Chem. Soc. 2009, 131, 1336.

[42] D. R. Dreyer, S. Park, C. W. Bielawski, R. S. Ruoff, Chem. Soc. Rev. 2010, 39, 228. 